BNL-113693-2017-JA

\title{
Self-Assembly of an $\alpha$-Helical Peptide into a Crystalline Two-Dimensional Nanoporous Framework
}

\author{
Elizabeth L. Magnotti, Spencer Hughes, Lillian Hough, Rebecca Dillard, \\ Arshad Karum-Bamkandathil, Tianquan Lian, Joseph P. Wall, \\ Xiaobing Zuo, Elizabeth Wright, Vincent P. Conticello
}

Submitted to Journal of the American Chemical Society

December 21, 2016

Biology Department

Brookhaven National Laboratory

\author{
U.S. Department of Energy \\ USDOE Office of Science (SC), \\ Biological and Environmental Research (BER) (SC-23)
}

Notice: This manuscript has been authored by employees of Brookhaven Science Associates, LLC under Contract No. DE- SC0012704 with the U.S. Department of Energy. The publisher by accepting the manuscript for publication acknowledges that the United States Government retains a non-exclusive, paid-up, irrevocable, world-wide license to publish or reproduce the published form of this manuscript, or allow others to do so, for United States Government purposes. 


\section{DISCLAIMER}

This report was prepared as an account of work sponsored by an agency of the United States Government. Neither the United States Government nor any agency thereof, nor any of their employees, nor any of their contractors, subcontractors, or their employees, makes any warranty, express or implied, or assumes any legal liability or responsibility for the accuracy, completeness, or any third party's use or the results of such use of any information, apparatus, product, or process disclosed, or represents that its use would not infringe privately owned rights. Reference herein to any specific commercial product, process, or service by trade name, trademark, manufacturer, or otherwise, does not necessarily constitute or imply its endorsement, recommendation, or favoring by the United States Government or any agency thereof or its contractors or subcontractors. The views and opinions of authors expressed herein do not necessarily state or reflect those of the United States Government or any agency thereof. 


\title{
Self-assembly of an $\alpha$-Helical Peptide into a Crystalline Two-Dimensional Nanoporous Framework
}

\author{
Elizabeth L. Magnotti, ${ }^{\dagger \perp}$ Spencer Hughes, ${ }^{\dagger}$ Lillian Hough, ${ }^{\dagger}$ Rebecca Dillard, ${ }^{\ddagger}$ Arshad Karum- \\ bamkandathil, ${ }^{\dagger}$ Tianquan Lian, ${ }^{\dagger}$ Joseph P. Wall, ${ }^{\S}$ Xiaobing Zuo, ${ }^{\natural}$ Elizabeth Wright, ${ }^{\ddagger}$ Vincent P. \\ Conticello* \\ ${ }^{\dagger}$ Department of Chemistry, Emory University, Atlanta, Georgia 30322, United States \\ * Department of Pediatrics, Emory University School of Medicine, Children's Healthcare of Atlanta, Atlanta, Georgia \\ 30322, United States \\ ${ }^{\S}$ Brookhaven National Laboratory, P.O. Box 500o, Upton, New York 11973, United States \\ " X-ray Science Division, Argonne National Laboratory, 970o South Cass Avenue, Argonne, Illinois 60439, United \\ States
}

KEYWORDS. Peptide design, self-assembly, nanosheet, porous material, honeycomb structure.

\begin{abstract}
Sequence-specific peptides have been demonstrated to self-assemble into structurally defined nano-scale objects including nanofibers, nanotubes, and nanosheets. The latter structures display significant promise for the construction of hybrid materials for functional devices due to their extended planar geometry. Realization of this objective necessitates the ability to control the structural features of the resultant assemblies through the peptide sequence. The design of a amphiphilic peptide, 3 FD-IL, is described that comprises two repeats of a canonical 18 amino acid sequence associated with straight $\alpha$-helical structures. Peptide 3FD-IL displays three-fold screw symmetry in a helical conformation and self-assembles into nanosheets based on hexagonal packing of helices. Biophysical evidence from TEM, cryo-TEM, SAXS, AFM, and STEM measurements on the 3 FD-IL nanosheets support a structural model based on a honeycomb lattice, in which the length of the peptide determines the thickness of the nanosheet and the packing of helices defines the presence of nanoscale channels that permeate the sheet. The resultant $2 \mathrm{D}$ materials may have potential as materials for nanoscale transport and controlled release applications.
\end{abstract}

\section{INTRODUCTION}

The creation of functional materials using the basic principles of molecular design is a main objective of the emergent field of nanoarchitectonics. ${ }^{1-2}$ Peptides, proteins, and related foldamers represent useful substrates for the construction of functional materials in that intermolecular interactions can be encoded within the corresponding sequence to direct self-assembly into well-defined supramolecular structures. Two-dimensional nano-scale assemblies (i.e., nanosheets) represent particularly desirable targets, in that these materials can serve as scaffolds to spatially arrange exogenous substrates, potentially with high precision, for integration into devices. To date, collagen-mimetic peptides, ${ }^{3-4}$ peptoids, ${ }^{5} \beta$-sheet peptides, ${ }^{6-7}$ and globular proteins ${ }^{8-9}$ have been employed for the creation of two-dimensional materials through self-assembly in solution.

In contrast, $\alpha$-helical peptides have not been utilized for fabrication of structurally defined two-dimensional assemblies. However, the crystallographically determined structures of synthetic helical peptides often reveal the presence of two-dimensional layers within threedimensional crystals, indicating the potential for nanosheet formation from self-assembly of helices. ${ }^{10-12}$ Similarly, uniformly sized rods of $\alpha$-helical poly(benzyl-Lglutamate) were observed to pack into layered structures of defined width in lyotropic mesophases and thin solid films. ${ }^{13}$ The thickness of the smectic layers could be directly correlated with the length of the peptide in an $\alpha$ helical conformation, and, therefore, is amenable to sequence control.

While helix-helix packing interactions are reasonably well understood from structural analyses of native proteins, ${ }^{14}$ most helix-helix interactions involve crossing angles that deviate from a strictly parallel packing arrangement. Classical coiled-coils, ${ }^{15}$ which comprise discrete bundles of $\alpha$-helices having a canonical heptad repeat sequence, display a crossing angle of approximately $20^{\circ}$ that defines a left-handed super-helical twist. However, the computational design of an antiparallel trimeric helical bundle was recently reported in which the $\alpha$-helices 
pack in a stable arrangement with minimal super-helical twist. ${ }^{16}$ These results suggest that straight $\alpha$-helices could form stable assemblies if the interacting helical faces were based on a repeat sequence that reinforced the canonical 18 amino acid repeat. ${ }^{17}$ We report herein the design of a synthetic peptide 3 FD-IL that self-assembles from aqueous solution to form structurally defined two-dimensional assemblies that comprise a hexagonal honeycomb structure based on packing of aligned straight $\alpha$-helices.

A abcdefghijklmnopqrabcdefghijklmnopqr AC-EALEKIAALEKIAALEKIAALKE IAALKEIAALKEIAK- $\mathrm{NH}_{2}$

B
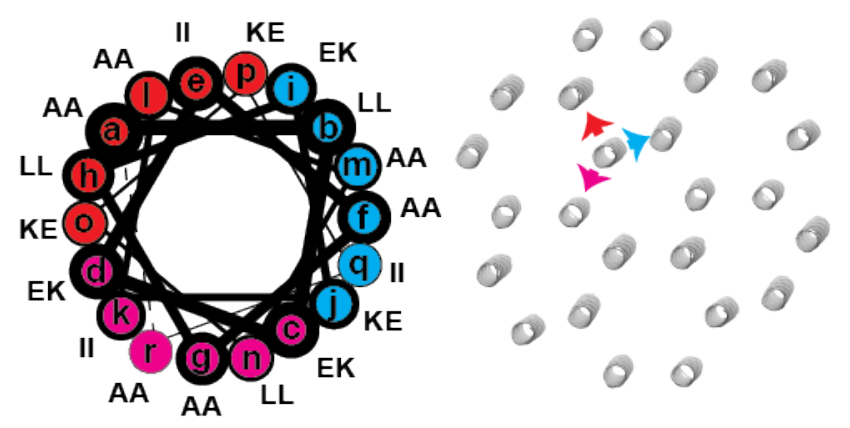

Figure 1. (A) Amino acid sequence of 3 FD-IL indicating the register of residues for the eighteen amino acid repeats. (B) Helical wheel representation of the repeat sequences of ${ }_{3}$ FDIL. (C) Proposed packing of 3 FD-IL helices within the hexagonal honeycomb model depicting the three-fold symmetry of the sequence. The three faces are represented in cyan, red, and magenta.

\section{RESULTS AND DISCUSSION}

Peptide 3FD-IL, a 38 amino acid sequence, was designed to form a straight $\alpha$-helix with three-fold screw symmetry (Figure $1 \mathrm{~A}$ ). The core peptide sequence (Ala2Ala37) consists of two successive 18 amino acid repeats; each of which defines three identical faces of six amino acids within the structural context of an $\alpha$-helical conformation (Figure $1 \mathrm{~B}$ ). Based on the geometrical parameters of an $\alpha$-helix, successive faces within the peptide sequence are angularly offset by $-120^{\circ}$ and axially offset by 9 $\AA ̊$ ( 6 x 1.5 Å helical rise/residue). ${ }^{17}$ In addition, the charged amino acids in the $\mathrm{N}$-terminal 3 faces are electrostatically complementary to the $C$-terminal 3 faces. Finally, a negatively charged glutamic acid residue and positively charged lysine residue were placed at the $N$-terminus and $C$-terminus of the capped peptide, respectively, to neutralize the structural influence of the helix macro-dipole. ${ }^{18}$

We hypothesized that the straight helices of ${ }_{3}$ FD-IL would behave as three-fold symmetric tectons that could self-assemble into a two-dimensional open framework structure having hexagonal symmetry. The $2 \mathrm{D}$ framework would be stabilized through a combination of hydrophobic interactions and electrostatic interactions between structurally adjacent helices. (Figure $1 \mathrm{~B}, 1 \mathrm{C}$ ). This mode of assembly should result in the creation of a nanoporous two-dimensional structure in which the side-chains of the charged amino acid side-chains line the lumen of the channel. (Figure $1 \mathrm{C}$ ).
A

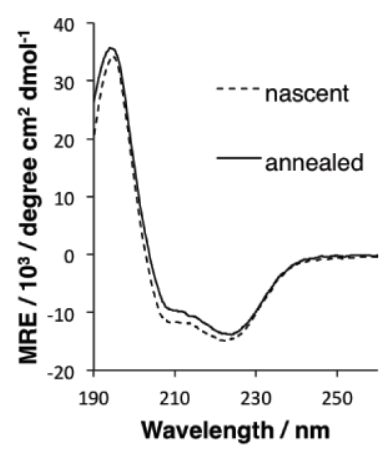

B

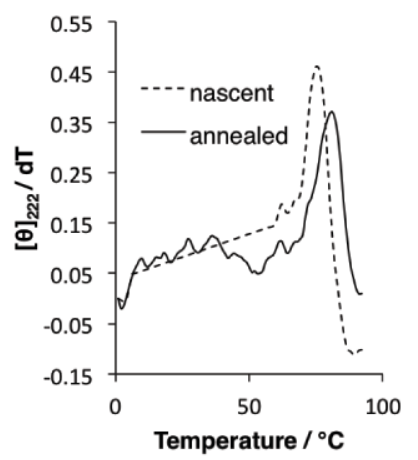

Figure 2. (A) Circular dichroism spectra of nascent and thermally annealed specimens of ${ }_{3}$ FD-IL in TAPS buffer (10 $\mathrm{mM}, \mathrm{pH}$ 8.5). (B) First derivative of the $\mathrm{CD}$ melting curves for nascent and thermally annealed solutions of 3 FD-IL in TAPS buffer (10 mM, pH8.5).

Peptide 3FD-IL could be readily prepared in high yield and purity via microwave assisted solid-phase peptide synthesis. The peptide was readily soluble in aqueous buffers within the $\mathrm{pH}$ range from 4-9. Circular dichroism spectropolarimetry indicated that 3 FD-IL adopted a strongly helical conformation within this $\mathrm{pH}$ range. The circular dichroism spectra of 3 FD-IL in aqueous buffer (10 mM TAPS, $\mathrm{pH}$ 8.5) are representative of its conformational properties and corresponded to conditions that promoted the formation of ordered assemblies (vide infra). CD spectra of nascent solutions of peptide 3 FD-IL indicated the presence of $\alpha$-helical secondary structure (Figure $2 \mathrm{~A}$ ). Thermolysis and slow cooling from $90^{\circ} \mathrm{C}$ to $40^{\circ} \mathrm{C}$ $\left(0.2^{\circ} \mathrm{C} /\right.$ minute) resulted in retention of the helical $\mathrm{CD}$ signature of 3 FD-IL (Figure $2 \mathrm{~A}$ ). The first derivative of the $\mathrm{CD}$ melting curve of nascent and thermally annealed samples of 3FD-IL displayed a similar response, in which a melting transition $\left(T_{m}\right)$ was observed at elevated temperature (Figure $2 \mathrm{~B}$ and Supporting Figure $\mathrm{S}_{1}$ ). The $T_{m}$ for the thermally annealed specimen of 3 FD-IL occurs at circa $84{ }^{\circ} \mathrm{C}$, which is slightly higher than the corresponding $T_{m}$ of $78{ }^{\circ} \mathrm{C}$ observed for the nascent sample. This observation may be attributable to the larger size of the selfassembled species after controlled thermal annealing ( $v i-$ de infra).

The self-assembly behavior of 3FD-IL was analyzed using transmission electron microscopy (TEM) and atomic force microscopy (AFM). TEM analysis of thermally annealed solutions of 3 FD-IL indicated the presence of twodimensional assemblies having a size distribution from a few hundred nanometers to several micrometers in apparent width. (Figure 3A). The nanosheets occasionally displayed sharp edges, but seemed susceptible to frag- 
mentation from mechanical shearing and/or deposition
A

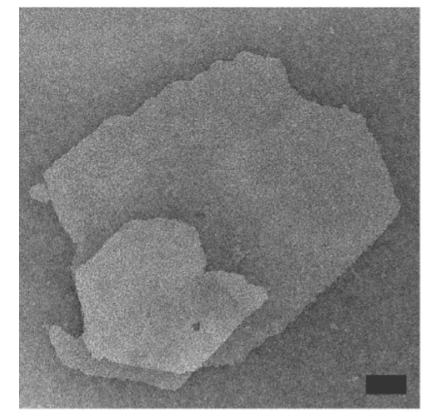

C

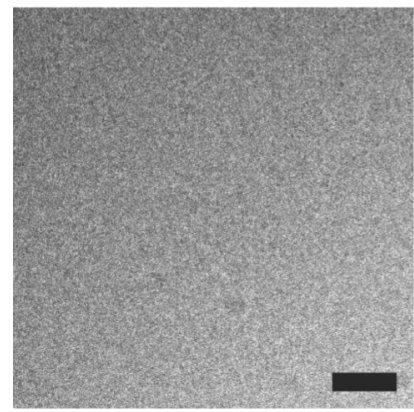

B

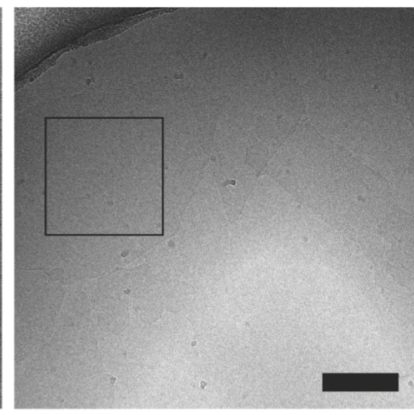

D

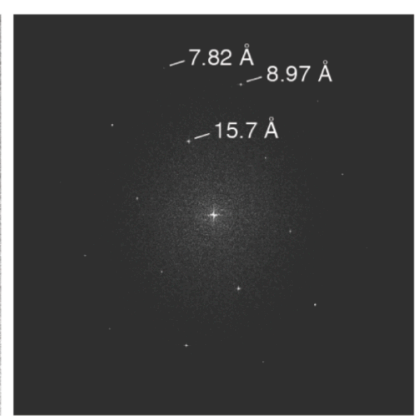

Figure 3. (A) TEM image of a representative nanosheet from self-assembly of annealed solutions of 3 FD-IL (scale bar $=200 \mathrm{~nm}$ ). (B) Cryo-TEM image of 3 FD-IL nanosheets (scale bar $=200 \mathrm{~nm})$. (C) Magnification of the boxed region displaying the periodic lattice of the 3 FD-IL nanosheet (scale bar $=50 \mathrm{~nm}$ ). (D) FFT of the boxed region indicating the hexagonal symmetry of the periodic lattice and associated lattice spacings.

on the EM grid. Note that TEM analysis of nascent solutions of 3 FD-IL indicated the presence of nanosheets, but these species were smaller than the corresponding assemblies from thermally annealed samples (Supporting Figure S2). The morphology of the 3FD-IL assemblies was confirmed using electron cryo-microscopy (Figure $3 \mathrm{~B}$ ), which demonstrated that the nanosheets remained largely intact under conditions of controlled vitrification. An enlargement of the lower magnification view (boxed region) revealed the presence of a periodic lattice within the nanosheet (Figure ${ }_{3} \mathrm{C}$ ). An FFT of this region displayed several lattice spacings, as well as the underlying hexagonal symmetry of the assembly (Figure 3D). In contrast, samples consisting solely of vitrified buffer lacked this lattice structure and its associated periodicity (Supporting Figure $\mathrm{S}_{3}$ ).

Tapping mode atomic force microscopy of the 3FD-IL assemblies confirmed the morphology observed from TEM measurements (Figure 4A). AFM measurements of the heights of the nanosheets indicated an apparent thickness of $62 \pm 1.6 \AA$ (Figure $4 \mathrm{~A}$ and $4 \mathrm{~B}$ ) with a relatively narrow distribution of heights and no evidence for multilayer sheets. The length of the peptide was calculated to be $57 \AA$ ( 38 residues $x 1.5 \AA$ rise/residue), assuming a uniform $\alpha$-helical conformation along the contour length of

the peptide. The close correspondence between the

A

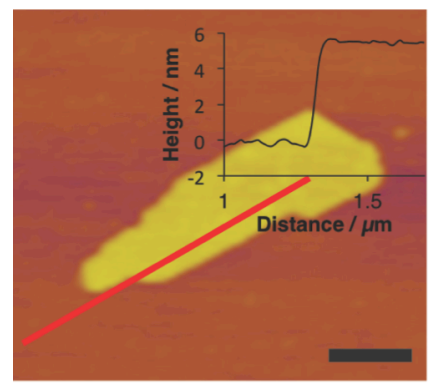

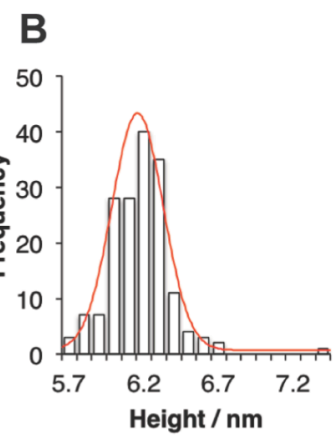

Figure 4. (A) Representative AFM image of 3FD-IL nanosheets with height profiles (inset). Scale bar $=200 \mathrm{~nm}$ (B) AFM height histogram with fit (red curve) for 3FD-IL nanosheets.

measured height of the nanosheet and the calculated length of ${ }_{3}$ FD-IL suggests that the peptides pack in a perpendicular orientation with respect to the surface of the nanosheet. We have observed a similar packing arrangement for nanosheets derived from collagen-mimetic peptides, in which the triple helices are oriented perpendicular to the sheet surface. ${ }^{3}$ The small discrepancy between the calculated and measured sheet thickness may reflect a slight registry shift between adjacent helices within the structure. Axial offsets of 2-3 $\AA$ are typically observed between anti-parallel helices in coiled-coil structures, ${ }^{19}$ and may be relevant to helix packing interactions within the structural model for the 3FD-IL nanosheets (vide infra).

Small angle X-ray scattering (SAXS) experiments were employed to determine information about the shape and internal structure of the assemblies in solution. A plot of intensity versus momentum transfer $(q)$ revealed several critical features related to the structure of the nanosheets. First, the scattering intensity in the low $q\left(\sim<0.05 \AA^{-1}\right)$ region followed a power law dependence of $q^{-2}$, indicating the presence of sheet-like or disk-like assemblies in solution (Figure $5 \mathrm{~A}$ and Supporting Figure $\mathrm{S}_{4}$ ). Second, fitting of the low $q$ data using the modified Guinier equation for sheet-like forms ${ }^{20}$ indicated that the sheets have an average thickness of circa $65 \AA$ in solution (Figure $5 \mathrm{~B}$ ), which agrees well with the AFM height measurements.

Furthermore, Bragg reflections were observed in the high $q$ region of the scattering curve (Figure $3 \mathrm{~A}$ ). This suggests that the nanosheets have a high degree of internal order. The Bragg reflections correspond to $d$-spacings of (1) $14.6 \AA$, (2) $8.4 \AA$, and (3) $7.3 \AA$. The relationship between these peaks is $(1):(1) / \sqrt{3}:(1) / \sqrt{4}$, which is consistent with a hexagonal arrangement of helices within the nanosheets. The FFT of the magnified image from electron cryo-microscopy displays the same mathematical relationship between the observed spacings, which occur at $15.7 \AA$, 9.0 $\AA$, and $7.8 \AA$ (Figure 3D). The slightly larger distances determined from cryo-EM may indicate that the lattice expands upon vitrification at low temperature. Nevertheless, the correspondence of structural parameters between measurements is consistent with formation 
of structurally defined populations of self-assembled nanosheets of 3FD-IL.

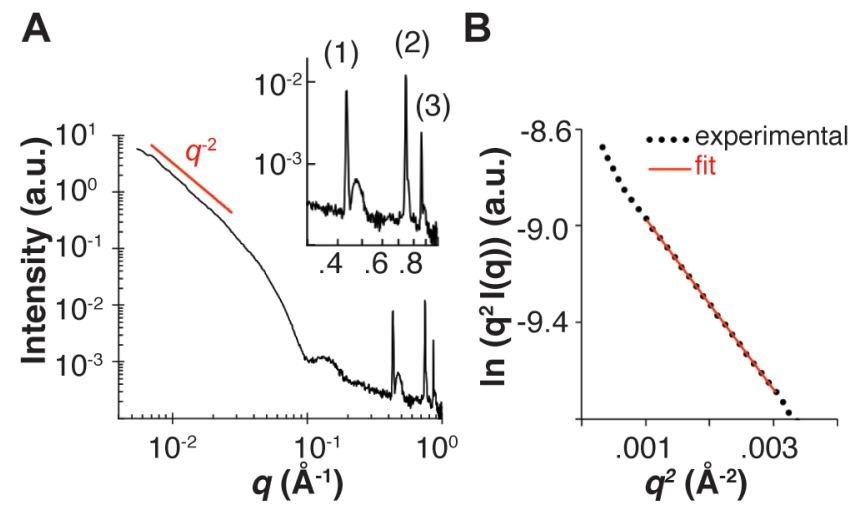

Figure 5. (A) Small-angle X-ray scattering (SAXS) curve for a solution of ${ }_{3}$ FD-IL $(10 \mathrm{mg} / \mathrm{mL})$ in TAPS buffer $(10 \mathrm{mM}, \mathrm{pH}$ 8.5). Inset: expansion of the high $q$ region depicting the positions of the three diffraction peaks. Peaks ( $d$-spacing): (1), $14.6 \AA$; (2), $8.4 \AA$; (3), $7.3 \AA$ A. (B) Modified Guinier plot, $\ln \left(q^{2}\right.$ $\mathrm{I}(q))$ versus $q^{2}$, of scattering data for 3 FD-IL using an equation for sheet-like forms: $q^{2} I(q)=I_{t, 0} \exp \left(-R_{t}^{2} q^{2}\right)$, where $I_{t, o}$ is a constant, and thickness $(T)$ can be calculated as $T=\sqrt{12} R_{t}$.

As the SAXS data were acquired at ambient temperature in buffered aqueous solution, we posited that these data were more representative of the structure under the conditions in which these materials might find application. Therefore, the distances from SAXS analysis were utilized to construct a structural model for the hexagonal unit cell. The two most probable packing arrangements of the $2 \mathrm{D}$ lattice would involve: (1) hexagonal close packing of helices or (2) a hexagonal honeycomb (i.e., graphenelike) structure in which helices are positioned at threefold sites in the $2 \mathrm{D}$ lattice. Pseudo-hexagonal close packing of helices has been commonly observed in the crystal structures of synthetic helical peptides ${ }^{10}$ as well as thin solid films from dried down lamellar mesophases of monodisperse, $\alpha$-helical PBLG. ${ }^{13}$ However, the three-fold screw symmetry of 3 FD-IL is more consistent with a honeycomb lattice (Figure 1). A detailed description of the two different models can be found in the Supporting Information (Supporting Figures $\mathrm{S}_{5}$ and S6).

The different structural models for the 3 FD-IL nanosheets were constructed on the basis of anti-parallel packing arrangements of adjacent helices based on several considerations. An anti-parallel orientation minimizes dipole-dipole repulsion between structurally adjacent $\alpha$ helices. ${ }^{18}$ In the crystal structures of helical peptides, antiparallel arrangements seem more common than parallel arrangements, probably due to the aforementioned consideration. ${ }^{10}$ However, the pattern of charged amino acids in the sequence of $\mathbf{3 F D}$ FIL is compatible with the formation of charge-complementary interactions in either a parallel or an anti-parallel alignment between helices.
To test for relative helix orientation, two peptide variants of 3 FD-IL were designed in which the sequences comprised different patterns of charged residues, but the overall amino acid composition and polar patterning were retained. Peptides 3FD-IL-EKEK and 3FD-IL-EEKK were designed such that the pattern of charged amino acids favored the parallel and anti-parallel packing arrangements, respectively (Supporting Figure $S_{7}$ ). The CD spectra of both peptides displayed a strongly $\alpha$-helical signature (Supporting Figure S8). However, TEM image analysis indicated that only 3 FD-IL-EEKK, the anti-parallel peptide, formed nanosheets (Supporting Figure S9). In contrast, TEM image analysis of assemblies derived from the parallel peptide, 3FD-IL-EKEK, formed fibrils (Supporting Figure S10). Moreover, synchrotron SAXS analysis of annealed nanosheet solutions of the anti-parallel peptide, 3FD-IL-EEKK, displayed a similar scattering profile to that observed for nanosheets of 3 FD-IL, including the position of the diffraction peaks in the high $q$ region of the scattering curve (Supporting Figure S11). These data indirectly suggest that adjacent helices within the 3 FD-IL nanosheets are aligned in an antiparallel orientation.



Figure 6. (A) STEM mass/area histogram for freeze-dried specimens of the 3 FD-IL nanosheets with Gaussian fits for a major (red) and minor (magenta) population of selfassembled species. (B) Comparison of the experimental scattering profile (orange curve) for 3 FD-IL nanosheets to a series of calculated scattering profiles for a hexagonal honeycomb structure in which the channels are empty (red curve), filled with water (blue curve), or filled within an additional helix (green curve). Calculated scattering curves were based on a disk-like model with a radius of $250 \AA$ using the lattice parameters derived from the original SAXS measurements.

Two lines of evidence were employed to distinguish between the hexagonal honeycomb and hexagonal closepacked structural models. Scanning transmission electron microscopy (STEM) was utilized to determine the mass/area (M/A) values for freeze-dried nanosheets of 3FD-IL. The histogram of the mass/area plot indicates the presence of a major species with a mean M/A value of 31.7 $\pm 1.4 \mathrm{Da} / \AA^{2}$ (Figure 6A) The theoretical mass-per-area measurement can be calculated for the two model lattices, which affords values of $33.2 \mathrm{Da} / \AA^{2}$ and $50.1 \mathrm{Da} / \AA^{2}$ for the hexagonal honeycomb and hexagonal close-packed lattices, respectively (Figure 7 and Supporting Figures $\mathrm{S}_{5}$ and S6). Therefore, the experimental STEM mass/area 
data more closely correspond to the hexagonal honeycomb model (Figure 7). A minor species with a higher mass/area $\left(38.8 \pm 3.6 \mathrm{Da} / \AA^{2}\right)$ is also observed, although it is less than that expected for the hexagonal close-packed lattice. FFT of STEM images obtained on negatively stained nanosheets of 3 FD-IL indicated the presence of a periodicity of 15.1 Å with hexagonal symmetry (Supporting Figure $\mathrm{S}_{12}$ ). The observed value from STEM data is slightly larger than the $14.6 \AA$ spacing from the SAXS data, but not significant enough to skew the conclusions of the analysis. These observed differences in lattice parameters might result from slightly different methods for sample preparation between the different analytical methods, which become manifested due to the porosity of the honeycomb structure.

A

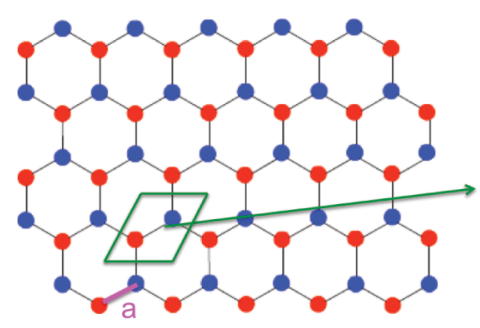

B

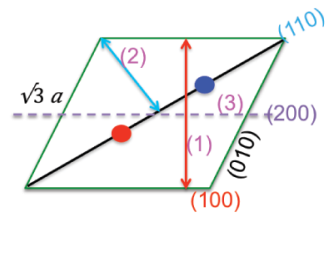

Figure 7. (A) Top view of the proposed hexagonal honeycomb lattice model for the 3 FD-IL nanosheets. Adjacent helices are aligned anti-parallel to each other with oppositely oriented helices depicted as red and blue circles. The unit cell is depicted in green. The inter-helical distance $(a)$ is calculated to be 9.7 $\AA$. (B) Expansion of the $2 \mathrm{D}$ unit cell of the ${ }_{3}$ FD-IL nanosheets. The lattice spacings corresponding to (1) $d_{100}(14.6 \AA),(2) d_{100}(8.4 \AA)$, and (3) $d_{200}(7.3 \AA)$ are indicated. The length of the $2 \mathrm{D}$ unit cell is $16.8 \AA(\sqrt{3} \times 9.7 \AA)$. The area of the parallelogram that defines the $2 \mathrm{D}$ unit cell is $245 \AA^{2}$ (base of $16.8 \AA \mathrm{x}$ height of $14.6 \AA$ ). The unit cell contains two peptides of mass $4070 \mathrm{Da}$. The calculated mass/area of the model structure is $33.2 \mathrm{Da} / \AA^{2}\left(2 \times 4070 \mathrm{Da} / 245 \AA^{2}\right)$.

In addition, theoretical modeling of the SAXS data, especially in the high $q$ region of the scattering profile, provided additional support for the hexagonal honeycomb model for the 3FD-IL nanosheets. The latter model requires the presence of internal nano-sized pores within the two-dimensional lattice (Figure $\mathrm{C}$ and Figure 7). In this model, the pores would be lined with polar amino acid residues. Theoretical modeling of SAXS profile of the honeycomb structure approximates quite well the experimental scattering curve. Modeling of water molecules into the channels does not significantly alter the theoretical scattering profile. However, additional peptides modeled into the channel (essentially equivalent to the hexagonal close-packed model) results in significant changes to the calculated scattering profile that do not replicate the experimental SAXS data. The absence of the sharp peak at a $q$ value of $0.431 \AA^{-1}$ (corresponding to the $d$-spacing of $14.6 \AA$ ) in the calculated scattering curve is quite distinct from that of the experimental scattering profile. Moreover, the honeycomb lattice is qualitatively in agreement with structural data on helix-helix interactions. The spacing between helices was determined to be $9.7 \AA$, which is consistent with the centroid-to-centroid distances between helices within crystal structures of helical peptides $^{10}$ and in $\alpha$-helical coiled-coils. ${ }^{21}$

Collectively, these data support a model for the 3 FD-IL nanosheets in which the helices are aligned perpendicular to the surface of the assembly and in which structurally adjacent helices are aligned in an anti-parallel orientation (Figure 7). Recent reports of nanosheets based on collagen-mimetic peptides have indicated similar arrangements of triple helices in a $2 \mathrm{D}$ lattice. ${ }^{3}$ The ${ }_{3}$ FD-IL nanosheets differ from the collagen nanosheets in that an open framework structure based on hexagonal packing symmetry is observed upon self-assembly. In contrast, the structural evidence supports tetragonal packing of collagen triple helices as a recurrent feature within the corresponding $2 \mathrm{D}$ assemblies.

Lanci, et al., have observed a honeycomb structure in a computationally designed peptide crystal, in which $2 \mathrm{D}$ layers of triple helical coiled-coils stack along the $\mathrm{z}$ direction (crystallographic c-axis). ${ }^{22}$ Single layers of the corresponding structures have not been observed, thus far, as isolated nanosheets in solution, perhaps due to relatively weak lateral interactions between trimers. More recently, the formation of a two-dimensional honeycomb structure was observed upon assembly of an angiotensinderived peptide on $\mathrm{Au}(111){ }^{23}$ However, the honeycomb structure formed on the substrate after electrospray ion beam deposition of the peptide from the gas phase under ultra-high vacuum conditions. From the discussion, it remains unclear whether similar species would assemble in solution or if the $2 \mathrm{D}$ layers could desorb to form freely standing assemblies with retention of the honeycomb architecture. $^{23}$

The hexagonal honeycomb morphology has been observed in layered structures corresponding to metalorganic frameworks (MOFs), ${ }^{24}$ covalent organic frameworks (COFs), ${ }^{25}$ and supramolecular organic frameworks (SOFs). ${ }^{26}$ However, the isolation of freely standing nanosheets of uniform thickness and internal structure from these materials represents a significant challenge. In the case of MOFs and COFs, individual layers may have a thickness of only one to a few atoms. Consequently, the formation of stable structures requires strong interactions between the supramolecular layers, which can be challenging to disrupt to form structurally uniform nanosheets that can be dispersed in solution. Nonetheless, significant progress has been made using soft exfoliation methods to afford nanosheets of one to a few layers in thickness. ${ }^{27}$

In contrast, supramolecular self-assembly in solution holds significant promise for fabrication of freely standing, structurally defined nanosheets. ${ }^{26-31}$ Self-assembly of two-dimensional assemblies having a hexagonal honeycomb structure was observed using molecular recognition 
of multi-valent guest-host species. $^{26}$ The 3FD-IL nanosheets more closely resemble these supramolecular organic frameworks (SOFs) than two-dimensional assemblies derived from either MOFs or COFs. The lamellar thickness, the internal structure, and the surface chemistry of the nanosheet can be controlled through the sequence and conformation of the peptide. DNA origami offers an even greater degree of structural control for the formation of $2 \mathrm{D}$ assemblies. ${ }^{32}$ However, recent studies on the de novo design of synthetic peptide assemblies have demonstrated progress toward the creation of ordered assemblies that approach the complexity of structures derived from DNA nanotechnology. .2,33-36 $^{-3}$

\section{CONCLUSIONS}

The formation of the $\mathbf{3}$ FD-IL nanosheets indicates that robust two-dimensional structures can result from selfassembly of simple $\alpha$-helical protomers. The corresponding nanosheets display significant long-range order and thermal stability at relatively low concentration (circa o.1$2.5 \mathrm{mM}$ ) in solution. The sequence of ${ }_{3}$ FD-IL directs the formation of a hexagonal honeycomb structure that defines nano-sized pores, which suggests that these materials may find application in transport and encapsulation of appropriately sized ions and small molecules. Further development of these materials for applications will require the availability of higher resolution (i.e., atomiclevel) structural information. Recently, direct electron detection devices, in combination, with electron cryomicroscopy have enabled reconstruction at near atomicresolution structures of natural ${ }^{37}$ and synthetic ${ }^{38}$ proteinand peptide-based helical assemblies and of $2 \mathrm{D}$ crystals of membrane proteins such as aquaporin-o (AQPo). ${ }^{39}$ The extended structural order of the 3FD-IL assemblies suggests that they might serve as useful substrates in this type of structural analysis, which would provide insights that could facilitate the design of more structurally complex and operationally functional $2 \mathrm{D}$ assemblies.

\section{METHODS}

Materials. Chemical reagents were purchased from Sigma Aldrich Chemical Co. (St. Louis, MO) or Anaspec, Inc. (Fremont, CA), unless otherwise noted. Fmoc-PEGPAL-PS resin for solid-phase peptide synthesis was purchased from Applied Biosystems, Inc. (Foster City, CA).

Peptide Synthesis. The ${ }_{3}$ FD-IL peptide and the ${ }_{3}$ FDIL-EKEK and 3FD-IL-EEKK variants were synthesized using microwave-assisted synthesis on a CEM Liberty solid-phase peptide synthesizer. Fmoc-PEG-PAL-PS resin was used for synthesis, which affords the $C$-terminal amide derivatives. Standard Fmoc protection chemistry was used for coupling, which was promoted using standard activation protocols based on DIEA/HBTU and baseinduced deprotection of the Fmoc group (20\% piperidine in dimethylformamide). All peptides were acetylated at the $N$-terminus using $20 \%$ acetic anhydride. The crude peptides were purified using reverse phase high performance liquid chromatography (HPLC) on a C18 column using a water-acetonitrile ( $0.1 \%$ trifluoroacetic acid) gra- dient. The target HPLC fractions were collected and lyophilized. The purity of the peptides was analyzed using electrospray ionization mass spectrometry and analytical HPLC (see Supporting Figures S13-S18). The peptides 3FDIL, 3FD-IL-EKEK, and 3FD-IL-EEKK were dissolved in aqueous buffer (10 mM TAPS, $\mathrm{pH} 8.5$ ) at an approximate concentration of $10 \mathrm{mg} / \mathrm{mL}$. Thermal annealing of the peptide samples was performed using the following protocol in a programmable thermal cycler: (1) peptide solutions were initially heated to $90^{\circ} \mathrm{C}$ for ten minutes and (2) peptide solutions were cooled to a final temperature of $40{ }^{\circ} \mathrm{C}$ at a cooling rate of $0.2^{\circ} \mathrm{C} /$ minute. The resultant specimens were stored at ambient temperature prior to analysis.

Circular Dichroism Spectropolarimetry. CD measurements were performed on a Jasco J-810 CD spectropolarimeter in $0.10 \mathrm{~mm}$ quartz cells (Hellma Analytics) at a peptide concentration of $1 \mathrm{mg} / \mathrm{mL}$ in $10 \mathrm{mM}$ TAPS buffer ( $\mathrm{pH} 8.5$ ). Spectra were recorded from 260 to $190 \mathrm{~nm}$ at a scanning rate of $50 \mathrm{~nm} / \mathrm{min}$ and a resolution of $0.1 \mathrm{~nm}$. $\mathrm{CD}$ melting experiments were performed in the temperature range from $5{ }^{\circ} \mathrm{C}$ to $95{ }^{\circ} \mathrm{C}$ at a heating rate of 40 ${ }^{\circ} \mathrm{C} /$ hour. The intensity of the $\mathrm{CD}$ signal at $222 \mathrm{~nm}$ was monitored as a function of temperature. Melting temperatures were obtained from the first derivative of the melting curves.

Transmission Electron Microscopy. TEM specimens were prepared from aqueous solutions of 3 FD-IL (10 $\mathrm{mg} / \mathrm{mL}$ ) and the ${ }_{3}$ FD-IL-EKEK and 3 FD-IL-EEKK variants in TAPS buffer (10 mM, pH 8.5) at the same concentration. The samples were deposited onto 200 mesh carbon-coated copper grids from Electron Microscopy Sciences (Hatfield, PA). After a 1 min incubation period, the samples were washed with $\mathrm{dd}_{2} \mathrm{O}$ and stained with an aqueous solution of phosphotungstate stain (0.1\%). Excess stain was wicked away from the grids after incubation on the grid for $30 \mathrm{~s}$. TEM measurements were acquired on a JEOL JEM-140o transmission electron microscope at an accelerating voltage of $120 \mathrm{kV}$.

Cryo-electron Microscopy. An aqueous solution of thermally annealed 3FD-IL nanosheets $(10 \mathrm{mg} / \mathrm{mL})$ in TAPS buffer $(10 \mathrm{mM}, \mathrm{pH} 8.5)$ was diluted 1:10 with TAPS buffer and utilized for cryo-electron microscopy. The nanosheets were diluted to an approximate concentration of 1 $\mathrm{mg} / \mathrm{mL}$ using TAPS buffer (10 mM, pH 8.5). Cryo-EM specimens were prepared by applying aliquots $(4 \mu \mathrm{l})$ of the diluted solution of nanosheets to glow-discharged 200 mesh copper Quantifoil grids (Quantifoil Micro Tools GMBH; Großlöbichau, Germany) and plunge freezing in liquid ethane using a Vitrobot Mark III (FEI, Hillsboro, Oregon). Cryo-EM data was collected using a JEOL JEM2200FS $200 \mathrm{kV}$ FEG-TEM with an in-column Omega energy filter (slit width $20 \mathrm{eV}$ ). Images were acquired on a Direct Electron, LP DE-20 direct electron detector (San Diego, CA) at a nominal magnification of $20,000 \times$ for a pixel size of 2.94 A.

Atomic Force Microscopy. Specimens for AFM measurements were prepared from aqueous solutions of ${ }_{3}$ FDIL nanosheets that had been previously assembled in 
TAPS buffer (10 mM, pH 8.5) at a peptide concentration of $10 \mathrm{mg} / \mathrm{mL}$ solutions. The nanosheet solution was diluted to a peptide concentration of $0.25 \mathrm{mg} / \mathrm{mL}$ using TAPS buffer (o,5 mM, pH 8.5) immediately before deposition onto a freshly cleaved mica substrate. The sample was spin-coated at a rate of $1500 \mathrm{rpm}$ for $1 \mathrm{~min}$. The AFM experiments were performed using a MFP-3D-BIO from Asylum Research. Silicon AFM tips (MikroMasch U.S.A.; Watsonville, CA) with a force constant $(5.4-16 \mathrm{~N} / \mathrm{m})$ were used to image the specimens in tapping mode at a scan rate of $1 \mathrm{~Hz}$ with 256 points and lines.

Small- and Wide-Angle X-ray Scattering Measurements. Synchrotron SAXS/WAXS measurements were performed at the 12-ID-B beamline of Advanced Photon Sources at Argonne National Laboratory, using the methods described previously. ${ }^{3}$ Thermally annealed specimens of 3 FD-IL nanosheets were assembled in TAPS buffer (10 $\mathrm{mM}, \mathrm{pH} 8.5$ ) at a peptide concentration of $10 \mathrm{mg} / \mathrm{mL}$. The initial specimens were dialyzed against a solution of freshly prepared TAPS buffer (10 $\mathrm{mM}, \mathrm{pH} 8.5$ ) to remove residual trifluoroacetic acid. SAXS/WAXS data were acquired on the dialyzed solutions of peptide nanosheets in TAPS buffer (10 mM, pH 8.5) at $25{ }^{\circ} \mathrm{C}$. A quartz capillary flow cell (1.5 mm diameter) was employed to prevent radiation damage. Twenty images were collected for each sample and buffer. The 2-D scattering images were converted to 1-D SAXS curves through azimuthally averaging after solid angle correction and then normalizing with the intensity of the transmitted x-ray beam, using the software package at beam-line 12ID-B. The 1-D curves of the samples were averaged and subtracted with the background measured from the corresponding buffers.

Scanning Transmission Electron Microscopy. STEM data were acquired at Brookhaven National Laboratory (BNL). The STEM instrument operates at $40 \mathrm{keV}$ with a scanning probe of $<0.3 \mathrm{~nm}$ diameter produced from a cold field-emission source. Every electron emerging from the specimen is detected by one of the scintillatorphotomultiplier detectors collecting 0-15 mRadian (bright field), 15-40 mRadian (small-angle dark field) and 40-200 mRadian (large-angle dark field). The large-angle signal is proportional to the mass of atoms in the path of the beam. Specimen quality and mass calibration are checked by detailed comparison of the image to the known structure of tobacco mosaic virus (TMV). For mass-per-area (M/A) measurements, TMV rafts at a theoretical M/A value of $81.9 \mathrm{Da} / \AA^{2}$ were employed for calibration.

Thermally annealed specimens of 3 FD-IL nanosheets were assembled in TAPS buffer (10 mM, pH 8.5) at a peptide concentration of $10 \mathrm{mg} / \mathrm{mL}$ solutions. The initial specimens were diluted to a concentration of $3 \mathrm{mg} / \mathrm{mL}$ and dialyzed against a solution of freshly prepared TAPS buffer (10 mM, pH 8.5). Specimens are deposited on thin carbon (circa $2 \mathrm{~nm}$ thick) supported on a thicker holey carbon film mounted on a titanium grid using the wetfilm, hanging-drop method. ${ }^{40}$ TMV is added to the grid first as an internal control, followed by injection buffer, then specimen solution (in $20 \mathrm{mM}$ MOPS buffer, $\mathrm{pH}$ 7.0) for $1 \mathrm{~min}$, then 10 washes of $20 \mathrm{mM}$ ammonium acetate $\mathrm{pH}$ 7.o. Excess solution is wicked from the edge with filter paper between each injection. After the last wash the grid is wicked to a thin layer (circa $1 \mathrm{~mm}$ ), fast frozen by plunging into liquid nitrogen shush and stored under liquid nitrogen. Grids are freeze-dried overnight in an ion-pumped chamber with an efficient cold trap and transferred under vacuum to the STEM cold stage $(-160$ ${ }^{\circ} \mathrm{C}$ ). Imaging typically uses a dose of $20 \mathrm{el} / \AA^{2}$ (causing < $5 \%$ mass loss, corrected by comparison to TMV). Mass measurements were performed off-line with the customized software PCMass. ${ }^{41}$

\section{ASSOCIATED CONTENT}

\section{Supporting Information}

Additional experimental characterization of peptides and peptide assemblies is available free of charge as a pdf file via the Internet at http://pubs.acs.org.

\section{AUTHOR INFORMATION}

\section{Corresponding Author}

"vcontic@emory.edu

\section{Present Addresses}

${ }^{\perp}$ Department of Cancer Immunology \& Virology, DanaFarber Cancer Institute; Department of Medicine, Harvard Medical School, 450 Brookline Avenue, Boston, Massachusetts 02215, USA

\section{Author Contributions}

The manuscript was written through contributions of all authors. All authors have given approval to the final version of the manuscript.

\section{Funding Sources}

V.P.C. acknowledges financial support from NSF grants CHE1012620 and CHE-141258o. T.L. acknowledges the financial support from the National Science Foundation (CHE1309817).

\section{Notes}

The authors declare no competing financial interest.

\section{ACKNOWLEDGMENT}

The electron microscopy data described here was gathered on either a JEOL JEM-220oFS 20okV TEM (supported by a National Science Foundation Major Research Instrumentation Grant 0923395) or a Hitachi HT7700 12okV TEM (supported by the Atlanta Clinical and Translational Science Institute (ACTSI) under award number ULiTRooo454). This study was supported in part by the Robert P. Apkarian Integrated Electron Microscopy Core (RPAIEMC), which is subsidized by the Emory College of Arts and Sciences and the Emory University School of Medicine and is one of the Emory Integrated Core Facilities. Additional support was provided by the National Center for Advancing Translational Sciences of the National Institutes of Health under award number ULiTRooo454. The content is solely the responsibility of the authors and does not necessarily reflect the official views of the National Institutes of Health. This work benefit- 
ed from the use of the A.P.S. funded by U.S. D.O.E. Office of Basic Energy Sciences, Division of Material Sciences, under contract W-31-109-Eng-38. We acknowledge the assistance of Dr. Anil Mehta for the construction of the molecular model of the honeycomb structure.

\section{REFERENCES}

(1) Ariga, K.; Li, J.; Fei, J.; Ji, Q.; Hill, J. P. Adv. Mater. 2016, 28, 1251-1256.

(2) Govindaraju, T.; Avinash, M.B. Nanoscale 2012, 4, 61026117.

(3) (a) Jiang, T.; Xu, C.; Liu, Y.; Liu, Z.; Wall, J. S.; Zuo, X.; Lian, T.; Salaita, K.; Ni, C.; Pochan, D.; Conticello, V. P. J. Am. Chem. Soc. 2014, 136, 4300-4308. (b) Jiang, T.; Xu, C.; Zuo, X.; Conticello, V. P. Angew. Chem., Int. Ed. 2014, 53, 8367-8371. (c) Jiang, T,; Vail, O.A.; Jiang, Z.; Zuo, X.; Conticello, V.P. J. Am. Chem. Soc. 2015, 137, 7793-7802.

(4) (a) Xu, F.; Khan, I.J.; McGuinness, K.; Parmar, A.S.; Silva, T.; Murthy, N.S.; Nanda, V. J. Am. Chem. Soc. 2013, 135, 1876218765. (b) Parmar, A.S.; James, J.K.; Grisham, D.R.; Pike, D.H.; Nanda, V. J. Am. Chem. Soc. 2o16, 138, 4362-4367.

(5) (a) Nam, K.T.; Shelby, S.A.; Choi, P.H.; Marciel, A.B.; Chen, R.; Tan, L.; Chu, T.K.; Mesch, R.A.; Lee, B.C.; Connolly, M.D.; Kisielowski, C.; Zuckermann, R.N. Nat. Mater. 2010, 9, 454-46o. (b) Sanii, B.; Kudirka, R.; Cho, A.; Venkateswaran, N.; Olivier, G.K.; Olson, A.M.; Tran, H.; Harada, R.M.; Tan, L.; Zuckermann, R.N. J. Am. Chem. Soc. 2011, 133, 20808-20815. (c) Kudirka, R.; Tran, H.; Sanii, B.; Nam, K.T; Choi, P.H.; Venkateswaran, N.; Chen, R.; Whitelam, S.; Zuckermann, R.N. Biopolymers 2011, 96, 586-595. (d) Robertson, E.J.; Olivier, G.K.; Qian, M.; Proulx, C.; Zuckermann, R.N.; Richmond, G.L. Proc. Natl. Acad. Sci. U.S.A. 2014, 111, 13284-13289.

(6) Hamley, I.W.; Dehsorkhi, A.; Castelletto, V. Chem. Commun. 2013, 49, 1850-1852.

(7) Dai, B.; Li, D.; Xi, W.; Luo, F.; Zhang, X.; Zou, M.; Cao, M.; Hu, J.; Wang, W.; Wei, G.; Zhang, Y.; Liu, C. Proc. Natl. Acad. Sci. U.S.A. 2015, 112, 2996-3001.

(8) Suzuki. Y.; Cardone, G.; Restrepo, D.; Zavattieri, P.D.; Baker, T.S.; Tezcan. F.A. Nature 2016, 533, 369-373.

(9) (a) Brodin, J.D.; Ambroggio, X.I.; Tang, C.; Parent, K.N.; Baker, T.S.; Tezcan, F.A. Nat. Chem. 2o12, 4, 375-382. (b) Brodin, J.D.; Carr, J.R.; Sontz, P.A.; Tezcan, F.A. Proc. Natl. Acad. Sci. U.S.A. 2014, 111, 2897-2902.

(10) (a) Chothia, C.; Levitt, M.; Richardson, D. J. Mol. Biol. 1981, 145, 215-250. (b) Bowie, J.U. Nat. Struct. Biol. 1997, 4, 915917. (c) Walther D, Springer C, Cohen F.E. Proteins 1998, 33, 457459 .

(11) (a) Karle, I.L. Acta Crystallogr B. 1992, 48, 341-356. (b) Vasudev, P.G.; Shamala, N.; Balaram, P.G. J. Phys. Chem. B 20o8, 112, 1308-1314.

(12) (a) Privé, G.G.; Anderson, D.H.; Wesson, L.; Cascio, D.; Eisenberg, D. Protein Sci. 1999, 8, 1400-1409. (b) Patterson, W.R.; Anderson, D.H.; DeGrado, W.F.; Cascio, D.; Eisenberg, D. Protein Sci. 1999, 8, 1410-1422.

(13) Taylor, K.S.; Lou, M.Z.; Chin, T.M.; Yang, N.C.; Garavito, R.M. Protein Sci. 1996, 5, 414-421.

(14) (a) Yu, S.M.; Conticello, V.P.; Zhang, G.; Kayser, C.; Fournier, M.J.; Mason, T.L.; Tirrell, D.A. Nature 1997, 389, 167170. (b) Yu, S.M.; Soto, C.; Tirrell, D.A. J. Am. Chem. Soc. 2ooo, $122,6552-6559$.
(15) (a) Lupas, A.N,; Gruber, M. Adv. Protein Chem. 2005, 70, 37-78. (b) Woolfson, D.N.; Bartlett, G.J.; Bruning, M.; Thomson, A.R. Curr. Opin. Struct Biol. 2012, 22, 432-441.

(16) Huang, P.S.; Oberdorfer, G.; Xu, C.; Pei, X.Y.; Nannenga, B.L.; Rogers, J.M.; DiMaio, F.; Gonen. T.; Luisi, B.; Baker D. Science 2014, 346, 481-485.

(17) Pauling, L.; Corey, R.B.; Branson, H.R. Proc. Natl. Acad. Sci. U.S.A. 1951, 37, 205-211.

(18) Hol, W.J.G. Prog. Biophys. Molec. Biol. 1985, 45, 149-195.

(19) Grigoryan, G.; DeGrado, W.F. J. Mol. Biol. 2011, 405, 1079-1100.

(20) Porod, G. in Small Angle X-ray Scattering (Eds.: Glatter, O.; Kratky, O.), Academic Press, New York, 1982, pp. 17-51.

(21) Harbury, P.B.; Tidor, B.; Kim, P.S. Proc. Natl. Acad. Sci. U.S.A. 1995, 92, 8408-8412.

(22) Lanci, C.J.; MacDermaid, C.M.; Kang, S.G.; Acharya, R.; North, B.; Yang, X.; Qiu, X.J.; DeGrado, W.F.; Saven, J.G. Proc. Natl. Acad. Sci. U.S.A. 2012, 109, 7304-7309.

(23) Abb, S.; Harnau, L.; Gutzler, R.; Rauschenbach, S.; Kern, K. Nat. Commun. 2016, 7, 10335.

(24) Peng, Y.: Li, Y.; Ban, Y.; Jin, H.; Jiao, W.; Liu, X.; Yang, W. Science 2014, 346, 1356-1359.

(25) Bunck, D.N.; Dichtel, W.R. J. Am. Chem. Soc. 2013, 135, 14952-14955.

(26) (a) Zhang, K.D.; Tian, J.; Hanifi, D.; Zhang, Y.; Sue, A.C.; Zhou, T.Y.; Zhang, L.; Zhao, X.; Liu, Y.; Li, Z.T. J. Am. Chem. Soc. 2013, 135, 17913-17918. (b) Pfeffermann, M.; Dong, R.; Graf, R.; Zajaczkowski, W.; Gorelik, T.; Pisula, W.; Narita, A.; Müllen, K.; Feng, X. J. Am. Chem. Soc. 2015, 137, 14525-14532.

(27) Mitra, S.; Kandambeth, S.; Biswal, B.P.; Khayum M.A.; Choudhury, C.K.; Mehta, M.; Kaur, G.; Banerjee, S.; Prabhune, A.; Verma, S.; Roy, S.; Kharul, U.K.; Banerjee, R. J. Am. Chem. Soc. 2016, 138, 2823-2828.

(28) Kambe, T.; Sakamoto, R.; Hoshiko, K.; Takada, K.; Miyachi, M.; Ryu, J.; Sasaki, S.; Kim, J.; Nakazato, K.; Takata, M.; Nishihara, H. J. Am. Chem. Soc. 2013, 135, 2462-2465.

(29) (a) Bauer, T.; Zheng, Z. Renn, A. Enning, R.; Stemmer, A.; Sakamoto, J.; Schlüter, A.D. Angew. Chem. Int. Ed. 2011, 5o, 7879-7884. (b) Zheng, Z.; Opilik, L.; Schiffmann, F.; Liu, W.; Bergamini, G.; Ceroni, P.; Lee, L.; Schütz, A.; Sakamoto, J.; Zenobi, R.; VandeVondele, J.; Schlüter, A.D. J. Am. Chem. Soc. 2014, 136, 6103-6110. (c) Sakamoto, R.; Hoshiko, K.; Liu, Q.; Yagi, T.; Nagayama, T.; Kusaka, S.; Tsuchiya, M.; Kitagawa, Y.; Wong, W.; Nishihara, H. Nat. Commun. 2015, 6, 6713.

(30) (a) Zheng, Y.; Zhou, H.; Liu, D.; Floudas, G.; Wagner, M.; Koynov, K.; Mezger, M.; Butt, H.; Ikeda, T. Angew. Chem. Int. Ed. 2013, 52, 4845-4848. (b) Lee, E.; Kim, J. Lee, M. Angew. Chem. Int. Ed. 2009, 48, 3657-366o.

(31) Dong, R.; Pfeffermann, M.; Liang, H.; Zheng, Z.; Zhu, X.; Zhang, J.; Feng, X. Angew. Chem. Int. Ed. 2015, 54, 12058-12063.

(32) Rothemund, P. W. K. Nature 20o6, 440, 297-302.

(33) Gradišar, H.; Božič, S.; Doles, T.; Vengust, D.; HafnerBratkovič, I.; Mertelj, A.; Webb, B.; Šali, A.; Klavžar, S.; Jerala, R. Nat. Chem. Biol. 2013, 9, 362-366.

(34) (a) King, N.P.; Sheffler, W.; Sawaya. M.R.; Vollmar, B.S.; Sumida, J.P.; André, I.; Gonen, T.; Yeates, T.O.; Baker, D. Science 2012, 336, 1171-1174. (b) King, N.P.; Bale, J.B.; Sheffler, W.; McNamara, D.E.; Gonen, S.; Gonen, T.; Yeates, T.O.; Baker, D. Nature 2014, 510, 103-108. (c) Bale, J.B.; Park, R.U.; Liu, Y.; Gonen, S.; Gonen, T.; Cascio, D.; King, N.P.; Yeates, T.O.; Baker, D. Protein Sci. 2015, 24, 1695-1701. 
(35) Fletcher, J.M.; Harniman, R.L.; Barnes, F.R.; Boyle, A.L.; Collins, A.; Mantell, J.; Sharp, T.H.; Antognozzi, M.; Booth, P.J.; Linden, N.; Miles, M.J.; Sessions, R.B.; Verkade, P.; Woolfson, D.N. Science 2013, 340, 595-599.

(36) Burgess, N.C.; Sharp, T.H.; Thomas, F.; Wood, C.W.; Thomson, A.R.; Zaccai, N.R.; Brady, R.L.; Serpell, L.C.; Woolfson, D.N. J. Am. Chem. Soc. 2015, 137, 10554-10562.

(37) (a) Hospenthal, M.K.; Redzej, A.; Dodson, K.; Ukleja, M.; Frenz, B.; Rodrigues, C.; Hultgren, S.J.; DiMaio, F.; Egelman, E.H.; Waksman, G. Cell 2016, 164, 269-278. (b) Sborgi, L.; Ravotti, F.; Dandey, V.P.; Dick, M.S.; Mazur, A.; Reckel, S.; Chami, M.; Scherer, S.; Huber, M.; Böckmann, A.; Egelman, E.H.; Stahlberg, H.; Broz, P.; Meier, B.H.; Hiller, S. Proc. Natl. Acad. Sci. U.S.A. 2015, 112, 13237-13242. (c) Kudryashev, M.; Wang, R.Y.; Brack- mann, M.; Scherer, S.; Maier, T.; Baker, D.; DiMaio, F.; Stahlberg, H.; Egelman, E.H.; Basler, M. Cell 2015, 160, 952-962.

(38) (a) Egelman, E.H.; Xu, C.; DiMaio, F.; Magnotti, E.; Modlin, C.; Yu, X.; Wright, E.; Baker, D.; Conticello, V.P. Structure 2015, 23, 280-289. (b) Wang, R.Y.; Kudryashev, M.; Li, X.; Egelman, E.H.; Basler, M.; Cheng, Y.; Baker, D.; DiMaio, F. Nat. Methods 2015, 12, 335-338.

(39) Stahlberg, H.; Biyani, N.; Engel, A. Arch. Biochem. Biophys. 2015, 581, 68-77.

(40) http://www.bnl.gov/biology/stem/SpecPrepDetails.asp.

(41) Wall, J. S.; Simon, M. N. Methods Mol. Biol. 2001, $148,589-601$. PCMass is available at ftp.stem.bnl.gov. 


$$
+\rightarrow \rightarrow
$$

